\title{
SIFT-MIC Based Matching Location Algorithm for Wire Bonding
}

\author{
Fanzhi Kong ${ }^{1}$, Xiao Xiao
}

\begin{abstract}
Computer vision inspection system is often applied in automatic wire bonding to ensure the location accuracy and speed, while feature selection affects the performance of inspection system. In this paper, a new feature extraction and matching location algorithm based on SIFT-MIC is proposed for wire bonding. The algorithm chooses MIC corners in the neighborhood of SIFT feature points to increase the robustness of feature extraction. Meanwhile, the selection of SIFTMIC feature points is before the generation of SIFT descriptors, to reduce the computation and improve the efficiency of feature extraction. The vector angle method is chosen to finish the matching location of the eye-point images and images to be matched. Experimental results show that SIFT-MIC algorithm is invariant to translation and rotation, and has high performance in the positioning accuracy and detection efficiency. The proposed algorithm is suitable for MEMS wire bonding images with rich points and lines features.
\end{abstract}

Keywords: Wire bonding · SIFT. MIC · Vector angle method

\section{Introduction}

Computer vision inspection is a new testing technique which is based on the study of computer vision. And computer vision inspection is the kernel technology of the automatic wire bonding. Each step of wire bonding process involves position location in order to ensure bonding precision. The main functions of computer vision inspection system are to obtain the lead-frame and chip images by CCD automatically [1-3], and measure the position deviation between chip and leadframe using image processing algorithms for accurate positioning.

With the development of microelectronics products, especially micro-system technology, the characteristic dimensions of chips and MEMS products are decreasing and the structure is becoming more complex.

To satisfy the need of the small batch and variety of MEMS device package, the precision of the bonding device is kept increasing while the automation of the wire bonding device must also be improved.

So the research and improvement of vision inspection system has become the

\footnotetext{
${ }^{1}$ Fanzhi Kong

School of Electronic Information, Zhejiang University of Media and Communications,

Hangzhou, Zhejiang, China

e-mail: kfz3042@126.com

Xiao Xiao

School of Electronic Information, Zhejiang University of Media and Communications, Hangzhou, Zhejiang, China

e-mail: 489502618@qq.com
} 
key technology for better performance of wire bonding. The application of vision inspection system can improve the speed, positioning accuracy, stability of systems and processes of the IC and MEMS production, and can reduce the production cost and has important practical significance. Based on the deep analysis of wire bonding vision inspection, a SIFT-MIC based detection and positioning algorithm is implemented in this paper for wire bonding location.

\section{SIFT Descriptor}

Scale invariant feature transform (SIFT) [4] is a scale space based feature matching algorithm, which has stable feature extraction and good matching performance. SIFT algorithm is invariant to image translation, scaling, rotation and noise, which can be applied to compute the position parameters under the circumstance of rotation.

SIFT operator detects extreme points in scale space, calculates its precise location and constructs the feature vector to finish image matching. The construction of feature vector consists of the following four steps [5]: detection of extreme points in scale space; precise positioning of extreme points; direction parameters calculation of extreme points; formation of feature points descriptors. SIFT operator has good performance while extracting local image features, has a relatively high precision.

But the SIFT operator also has its disadvantages, such as large amount of calculation, poor real-time performance, and the correctly feature points matching rate is relatively low. With the decrease in size and structure complexity of MEMS products, the location algorithm of wire bonding vision inspection is required to achieve higher accuracy and real-time performance. The abundant lines and points feature of MEMS images should also be utilized in vision inspection algorithm design.

Based on the deep analysis and simulation experiments, an improved feature points extraction algorithm is given in this paper. The proposed algorithm improves the significance of corner points by searching the MIC corners [6] in the neighborhood of the selected SIFT feature points. Meanwhile, the selection of SIFT-MIC corner points is accomplished before the generation of SIFT descriptors, thus the computation is reduced and the efficiency of the algorithm is improved.

\section{SIFT-MIC Based Matching Location Algorithm}

The realization steps of SIFT-MIC matching location algorithm are described detailed as follows:

1. Establish DoG (Difference of Gaussian) scale space and find the extreme points of DoG as feature points candidates.

2. Determine the location and scale of the feature points candidates by quadratic function fitting to achieve sub-pixel precision. The low contrast feature points candidates and unstable edge points should be filtered.

3. Select the MIC corner points in the obtained SIFT feature points. Since the Gaussian smoothing convolution operation and the approximation error of DoG, the feature point candidates and corner points are not in superposition completely. Thus the MIC corner detection algorithm is applied in the neighborhood of the 
feature points candidates to search the optimal corner points.

In this paper, we set the radius of the neighborhood for detection as three pixels, and the detection performance comparison of SIFT-MIC and SIFT is illustrated in Fig. 1.

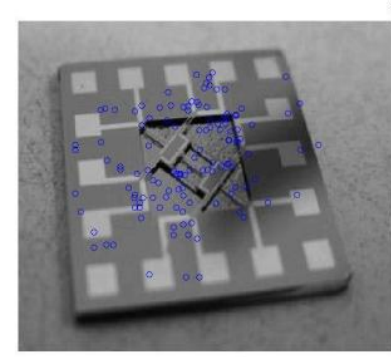

(a) SIFT-MIC

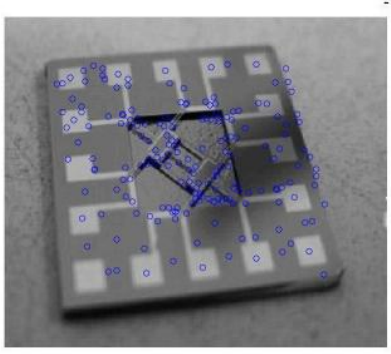

(b) SIFT

Fig. 1 Performance comparison of SIFT-MIC and SIFT algorithms

4. Compute the feature description for SIFT-MIC feature points. First the orientation parameters of feature points are calculated, and then the gradient direction histogram descriptor of the neighborhood is also obtained.

5. Finish the similarity measure of feature points sets. Generally, SIFT matching algorithm applies distance functions to determine the similarity between images, and the minimum distance is chosen as the optimal matching. Minimum distance method is very simple but the amount of calculation is intensively large. To meet the real-time requirement of the wire bonding vision system, the vector angle method is applied as the similarity measure between images to improve the matching speed.

In the process of computing vector angle, KD-Tree algorithm is chosen to build index for all elements of the testing image. Each feature point in eye-point image is also selected to compute its corresponding two optimal matching feature points with Best-Bin-First searching scheme.

6. Eliminate the outliers and the error matching points. False matches will inevitably be produced within the candidate matches obtained by similarity measure, and random sample consensus (RANSAC) algorithm is used to eliminate outliers. The error matching points are removed according to the geometric constraints and other additional matching constraints and the robustness of the SIFT-MIC algorithm is then improved.

7. Calculate the position parameters of the test wire bonding images. Singular Value Decomposition (SVD) method[7,8] is utilized according to the obtained SIFT-MIC feature points.

Let $A=\left\{a_{i}\right\}$ and $B=\left\{b_{i}\right\}(i=0,1, \ldots n)$ be the matching points sets of eye-point image and test image, because the relationship between the eye-point image and the test image is rigid transformation, which means only the displacement and rotation of them change, then we can have the following equation:

$$
a_{i}=s R b_{i}+T
$$

Where the scale factor $\mathrm{s}$ is $1, \mathrm{R}$ is a $2 \times 2$ rotation matrix, and $\mathrm{T}$ is a $2 \times 1$ translation vector. 
We aim to find the optimal $\mathrm{R}$ and $\mathrm{T}$ to minimize the function $f(R, T)=\sum_{i=1}^{N}\left\|a_{i}-\left(R b_{i}+T\right)\right\|$. The SVD method can easily compute the optimal R and $\mathrm{T}$ parameter.

The realization flow chart of SIFT-MIC algorithm is shown in Fig. 2.

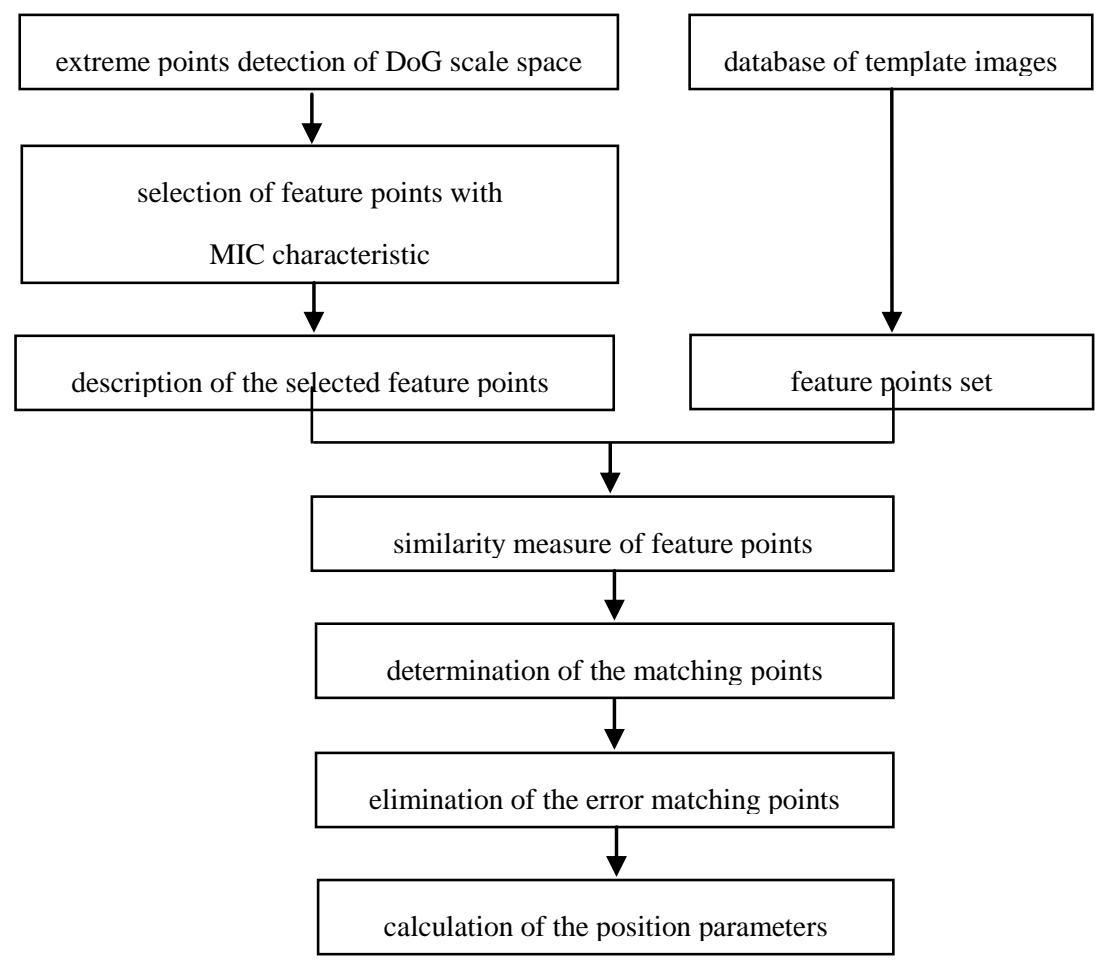

Fig. 2 Functional diagram of SIFT-MIC algorithm

\section{Experimental Results}

Simulation experiments were carried out to verify the effectiveness and performance of the proposed SIFT-MIC feature points extraction algorithm. The realization flow chart of the positioning algorithm is illustrated in Fig. 3.

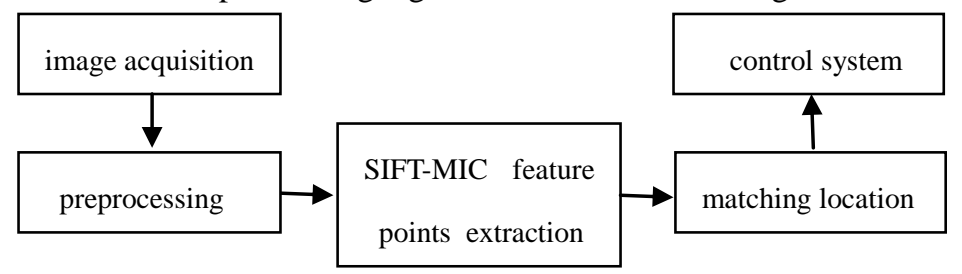

Fig. 3 SIFT-MIC based wire bonding location flow chart 
To test the invariance of the proposed algorithm, 50 MEMS images were transformed and two testing sets were given with different translation and rotation levels. Harris, SIFT and SIFT-MIC algorithms are applied to extract the feature points and compute the matching positions of the testing sets. The matching abilities and accuracy of Harris, SIFT and SIFT-MIC under different circumstances are compared and the results are illustrated in Fig. 4.

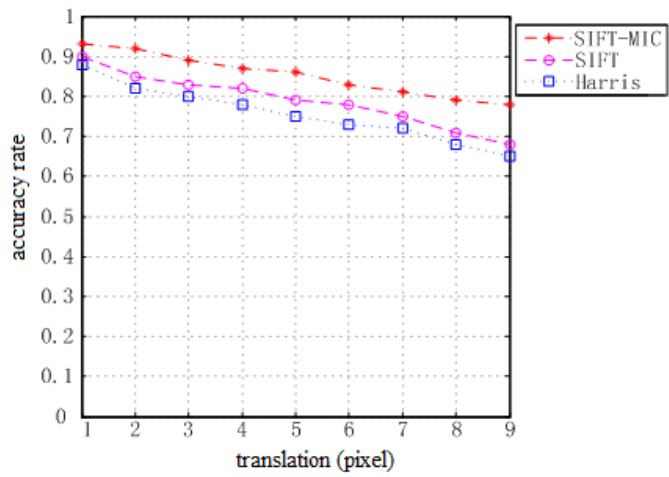

(a)

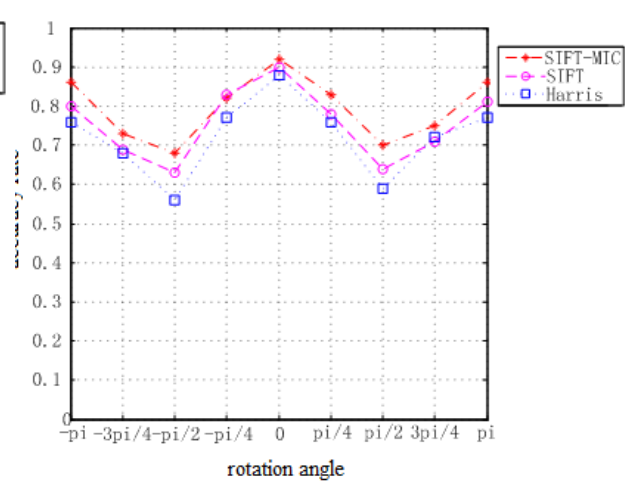

(b)

Fig. 4 Comparison of matching accuracy of three algorithms with translation and rotation

As can be seen from Fig. 4 (a) that the SIFT-MIC based matching location algorithm has higher accuracy rate compared with Harris and SIFT algorithm under the circumstance of translation. We can also see clearly from Fig. 4 (b) that the performance of the three algorithms declines consistently with the increase of rotation angle. The accuracy rate reaches a minimum with the rotation angle of $\pm \frac{\pi}{2}$, and the high accuracy in the case of $\pm \pi$ is due to the symmetry of chip images. In the process of the wire bonding, the rotation angle is usually very small and the positioning results will not be affected. We can also see that SIFT-MIC based algorithm is rotation invariant and has a higher accuracy.

To test the matching performance, MEMS images with rich points feature are chosen for wire bonding location experiments. All the eye-point images are preprocessed with normalized $64 \times 64$ picture size, and the effective searching region is $25 \%$ larger than the eye-point images. The combination of coarse searching and fine searching schemes is applied in the experiment to improve the matching efficiency and accuracy furthermore. According to the experiment results, the average positioning time and accuracy of SIFT-MIC, SIFT and the normalized grayscale correlation based location algorithms are shown in Table 1. 
Table1 Performance of SIFT-MIC based matching location algorithm

\begin{tabular}{|c|c|c|c|}
\hline \multirow{2}{*}{$\begin{array}{c}\text { matching location } \\
\text { algorithm }\end{array}$} & $\begin{array}{c}\text { average location time } \\
\text { (second) }\end{array}$ & \multicolumn{2}{|c|}{ average location accuracy } \\
\cline { 3 - 4 } & 1.842 & translation(pixel) & rotation(degree) \\
\hline SIFT-MIC & 2.563 & \pm 0.40 & \pm 0.3 \\
\hline SIFT & 6.870 & \pm 0.68 & \pm 0.5 \\
\hline $\begin{array}{c}\text { normalized grayscale } \\
\text { correlation }\end{array}$ & & \pm 1 & - \\
\hline
\end{tabular}

As can be seen from Table1, SIFT-MIC based location algorithm has superior positioning accuracy and detection efficiency in MEMS wire bonding visual location. Compared with the normalized grayscale correlation algorithm and the SIFT algorithm, the location accuracy and speed are improved. The proposed SIFT-MIC based algorithm can meet the requirement of wire bonding for real-time and accuracy, and can be well applied in MEMS images with detailed points feature.

\section{Conclusions}

Images matching location algorithm is especially important for computer vision inspection system, and the performance of location algorithm determines the speed and accuracy of computer vision inspection system. The selection of feature extraction algorithm is a core step in images matching location. The application of feature points in image location can decrease the amount of calculation involved without loss of image gray-level information.

In this paper, SIFT-MIC algorithm is given after the analysis of SIFT descriptor to increase the effectiveness of feature points. The proposed algorithm chooses the MIC corner in the neighborhood of SIFT feature points before generating SIFT descriptors, which reduces the computation and improve the efficiency of feature extraction. SIFT-MIC algorithm is also invariant to translation and rotation, and is suitable for MEMS wire bonding images which has rich points and lines features.

Experimental results show the SIFT-MIC based matching location algorithm has higher positioning accuracy and detection efficiency compared with the normalized grayscale correlation algorithm and the SIFT algorithm, and has important research and application value for wire bonding vision inspection system.

\section{References}

1. ESEC Co., "The image vision system of the full automatic wire bonder," Equipment for Electronic Products Manufacturing, vol.30(3), 2001, pp. 43-48.

2. Y.Hui, L. Xiaobin, G. Chen, "The calibration system design of wire bonder," Equipment for Electronic Products Manufacturing, vol. 34(12), 2005, pp. 30-33. 
3. S Kolloor. and S Balamurugan, "Machine vision for alignment and inspection in die bonder," Proc. SPIE, vol. 3185, 1997, pp.2-10.

4. Lowe D G. Object recognition from local scale-invariant features. Proc. IEEE Int. Conf Computer Vision, Kerkyra, Greece, Sept. 1999, 2:1150-1157P

5. Lowe D G. Distinctive image features from scale-invariant keypoints. International Journal of Computer Vision. 2004, 60(2): 91-110P

6. Trajkovic M and Hedley M. Fast corner detection. Image and Vision Computing, 1998, 16(1): 75-87P

7. Woods R P, Grafton S T, Holmes C J, et al. Automated image registration: General methods and intrasubject, intramodality validation. Journal of Computer Assisted Tomography. 1998 22(1): 139-152P

8. Michel A A, Frank P F, Terry M P. An algorithmic overview of surface registration techniques for medical imaging. Med Image Anal, 2000, 4: 201-217P

9. Foster III J W, Griffin P M, Messimer S L and Villalobos J R. Automated visual inspection: a tutorial. Computer and Industrial Engineering. 1990, 18(4):493-504P

10. Timothy S N, Anil K J. A Survey of Automated Visual Inspection. Computer Vision and Image Understanding. 1995, 61(2):231-262P

11. Gallegos-Hernandez, A, Ruiz-Sanchez F J and Villalobos-Cano J R. 2D automated visual inspection system for the remote quality control of SMD assembly. IECON Proc.. 2002(3):2219-2224P

12. Fanzhi Kong, Xingzhou Zhang, Yizhong Wang, Dawei Zhang \& Junlan Li, "Fast matching location algorithm based on mixed moment for wire bonding," 2008 International Conference on Optical Instrument and Technology. Beijing, China. Nov. 2008, Vol.7159, pp. 71590F$71590 \mathrm{~F}-9$. 